\title{
GMR
}

\section{CXCL12 G801A polymorphism and susceptibility to glioma: a case-control study}

\author{
S.F. Chang ${ }^{1 *}$, S.L. Li ${ }^{2 *}$, B. Yang', K.M. Yao ${ }^{2}$, R.H. Miao ${ }^{2}$, G.F. Liang ${ }^{2}$ and \\ K.M. Zhang ${ }^{2}$ \\ ${ }^{1}$ Department of Neurosurgery, The First Affiliated Hospital of Zhengzhou University, \\ Zhengzhou, Henan Province, China \\ 2Department of Neurosurgery, The Third People's Hospital of Luoyang, Luoyang, \\ Henan Province, China \\ *These authors contributed equally to this study. \\ Corresponding author: B. Yang \\ E-mail: boyangzzu@163.com
}

Genet. Mol. Res. 14 (4): 17399-17405 (2015)

Received August 13, 2015

Accepted October 11, 2015

Published December 21, 2015

DOI http://dx.doi.org/10.4238/2015.December.21.9

ABSTRACT. Previous studies have demonstrated that the CXCL12 G801A polymorphism is closely correlated with tumor susceptibility. In addition, the CXCL12/CXCR4 pathway is closely related to proliferation, metastasis, and invasion of glioma. However, the genetic effects of the CXCL12 G801A polymorphism on glioma risk in Chinese populations remain unknown. In this study, we investigated the potential associations between the CXCL12 G801A polymorphism with glioma susceptibility and its clinicopathological characteristics. Frequencies of CXCL12 G801A polymorphic variants between glioma patients $(N=750)$ and healthy controls $(N=750)$ were assessed using restriction length fragment polymorphism analysis. The association among the CXCL12 G801A polymorphism, glioma grade (WHO classification), and histological type was also evaluated. Our results showed that patients with glioma had significantly higher frequency of the CXCL12-3' A/A genotypes $(P=0.039)$ as compared with healthy controls. 
When stratified by the glioma histology, high-grade glioma patients had significantly higher frequency of the CXCL12-3' A/A genotypes $(P=0.019)$ as compared with low-grade glioma patients. When stratified by the WHO grade, significantly higher frequency of the CXCL12-3' A/A genotype was observed in stage IV glioma patients $(P=0.037)$. We conclude that the CXCL12 G801A polymorphism is a risk factor that increases susceptibility to gliomas in a subset of the general Han Chinese population.

Key words: CXCL12 G801A polymorphism; Glioma; Tumor susceptibility

\section{INTRODUCTION}

Gliomas are the most aggressive and common brain tumors in humans. Patients with gliomas usually have poor survival rates and unfavorable prognosis. Despite great progress in the area of neurosurgery and chemotherapy, the clinical outcomes of gliomas therapy is still unfavorable. Disease etiology of gliomas remains poorly understood. Gliomas may be caused by both intrinsic and environmental factors. Recently, a vast number of studies have shown that genetic factors such as single nucleotide polymorphisms (SNPs) play an important role in modifying glioma susceptibility (Feng et al., 2014).

CXCL12, also known as SDF-1, plays important roles in the regulation of hematopoietic cells' biological functions such as proliferation, differentiation, and migration (Bleul et al., 1999; Broxmeyer et al., 1999). In addition, CXCL 12 has been demonstrated to be closely correlated with tumor growth, angiogenesis, as well as progression and metastasis of various types of cancers (Balkwill 2003; Domanska et al., 2013). Studies have shown that CXCL12 can enhance the proliferation and invasion capacity of glioma cell lines (Zhang et al., 2005; do Carmo et al., 2010). In addition, glioma patients with positive CXCL12 expression have poorer prognosis (Salmaggi et al., 2005). A SNP (G to A) located at position 801 in an evolutionarily conserved segment of the 3'-untranslated region the CXCL12 gene has been showed to be associated with increased production of CXCL12 transcripts and proteins (Winkler et al., 1998; Garcia-Moruja et al., 2009). Moreover, CXCL12 G801A polymorphism has been linked to cancer susceptibility. A significant increase in the GA + AA genotype of the CXCL12 G801A polymorphism was observed in prostate cancer patients as compared with healthy controls. In addition, these patients with CXCL12 G801A polymorphism had greater lymph node metastasis (Hirata et al., 2007). Similarly, Razmkhah et al. (2005a,b) also showed that CXCL12 G801A polymorphism may be regarded as a risk factor for both lung cancer and breast cancer.

Although CXCL12 G801A polymorphism is closely correlated with tumor susceptibility, the genetic effects of CXCL12 G801A polymorphism on glioma risk in the Chinese populations remain unknown. Therefore, we investigated the association between CXCL12 G801A polymorphisms and glioma risk in the Chinese Han populations.

\section{MATERIAL AND METHODS}

\section{Study population}

This case-control study was approved by the ethnic committee of the First Affiliated Hospital of Zhengzhou University and the Third People's Hospital of Luoyang, and written inform consent 
was obtained from all subjects. Subjects were recruited from the Department of Neurosurgery, the First Affiliated Hospital of Zhengzhou University, and the Third people's Hospital of Luoyang between February 2012 to June 2014, which includes 750 glioma patients and 750 healthy controls. Control subjects were matched to glioma patients by age and sex. WHO criteria were employed to determine tumor type and stage. Smoking status was based on self-reports. Subjects who have never smoked or smoked less than 100 cigarettes in their lives were classified as non-smokers. Drinking status was defined as non-drinkers and drinkers (drinks per day). None of the glioma patients had suffered other cancers, and the healthy controls were cancer-free. All subjects were unrelated individuals of the Han Chinese. In addition, patients with glioma were pathologically confirmed. The clinical features of the study population are described in Table 1.

\begin{tabular}{|c|c|c|c|}
\hline Parameters & Cases $(N=750)$ & Controls $(N=750)$ & $P$ value \\
\hline \multicolumn{4}{|l|}{ Gender } \\
\hline Female & $364(48.53 \%)$ & $353(47.07 \%)$ & \\
\hline Male & $386(51.47 \%)$ & $397(52.93 \%)$ & 0.605 \\
\hline Age (years) & $56.3 \pm 15.6$ & $53.8 \pm 18.2$ & 0.365 \\
\hline \multicolumn{4}{|l|}{ Smoking status } \\
\hline Smoker & $292(38.93 \%)$ & $275(36.66 \%)$ & \\
\hline Non-smoker & $458(61.07)$ & $475(63.34 \%)$ & 0.394 \\
\hline \multicolumn{4}{|l|}{ Drinking status } \\
\hline Drinker & $160(21.33 \%)$ & $142(15.87 \%)$ & \\
\hline Non-drinker & $590(78.67 \%)$ & $608(84.13 \%)$ & 0.274 \\
\hline \multicolumn{4}{|c|}{ Family history of cancer } \\
\hline Yes & $108(14.40 \%)$ & $92(12.27 \%)$ & \\
\hline No & $642(85.60 \%)$ & $658(87.73 \%)$ & 0.471 \\
\hline \multicolumn{4}{|l|}{ Histology types* } \\
\hline High-grade glioma & $256(34.13 \%)$ & - & \\
\hline Low-grade glioma & $494(65.87 \%)$ & - & \\
\hline \multicolumn{4}{|l|}{ WHO } \\
\hline 1 & $156(20.80 \%)$ & - & \\
\hline II & $172(22.93 \%)$ & - & \\
\hline III & $143(19.07 \%)$ & - & \\
\hline IV & $279(37.20 \%)$ & - & \\
\hline
\end{tabular}

*High-grade glioma (glioblastoma), low-grade glioma (astrocytoma, oligodendroglioma, mixed glioma, and other lowgrade glioma).

\section{DNA extraction and genotyping}

DNA was extracted from peripheral blood by salt extraction. The 3'-UTR CXCL12 G801A polymorphism was analyzed using PCR-RFLP assays, and primers are as follows: sense, 5'-CAGTCAACCTGGGCAAAGCC-3'; antisense, 5'-AGCTTTGGTCCTGAGAGTCC-3'. Cycling parameters used are as follows: 1 cycle of DNA denaturation at $94^{\circ} \mathrm{C}$ for $5 \mathrm{~min}$. This was followed by 30 cycles at $94^{\circ} \mathrm{C}$ for $1 \mathrm{~min}, 55^{\circ} \mathrm{C}$ for $1 \mathrm{~min}$, and $72^{\circ} \mathrm{C}$ for $2 \mathrm{~min}$. Final extension step was performed at $72^{\circ} \mathrm{C}$ for $5 \mathrm{~min}$. The PCR products were then digested with Mspl restriction endonuclease, and fragments were run on a $2 \%$ agarose gel, stained with ethidium bromide, and visualized under UV light. Three banding patterns were observed: a 302-bp band (undigested) for the AA genotype; 302-, 202-, and 100-bp fragments for the AG genotype, and two 202- and 100-bp bands for the GG genotype. Genotyping analysis was performed blind for quality control. 


\section{Statistical analysis}

Distribution of genotypes in cases and controls was tested for deviation from the Hardy-Weinberg equilibrium. We used the Fisher exact test to examine the differences in genotypic and allelic distribution between glioma patients and controls. The odds ratio (OR) and $95 \%$ confidence intervals (Cls) were also evaluated. Statistical analysis was conducted using the SPSS 21.0 package (SPSS Inc., Chicago, IL, USA). P $<0.05$ was considered to be statistically significant.

\section{RESULTS}

\section{Frequency of CXCL12-801 genotypes and alleles in glioma patients and controls}

Genotype frequency distribution was found to be in accordance with the Hardy-Weinberg equilibrium in both patient and control groups. As showed in Table 1, we did not observe a significant difference between the two groups based on gender $(P=0.605)$, age $(P=0.365)$, smoking status $(P=0.394)$, drinking status $(P=0.274)$ and family history of cancer $(P=0.471)$. Among the 750 glioma cases, 256 cases were classified as high-grade glioma. In these cases, 156 cases had WHO grade I glioma, 172 cases had WHO grade II glioma, 143 cases had WHO grade III glioma, and 279 cases had WHO grade IV glioma.

Patients with glioma had significantly higher frequency of the CXCL12-3' A/A genotypes $(\mathrm{OR}=1.44 ; 95 \% \mathrm{Cl}: 1.021-2.018 ; \mathrm{P}=0.039$ ) as compared with the healthy controls (Table 2$)$. When stratified by the glioma histology, high grade glioma patients had significantly higher frequency of the CXCL12-3' A/A genotypes $(\mathrm{OR}=1.59 ; 95 \% \mathrm{Cl}: 1.10-2.31 ; \mathrm{P}=0.019)$ than low grade glioma patients (Table 3). As shown in Table 4, when stratified by the WHO grade, significantly higher frequency of the CXCL12-3' A/A genotype was observed in stage IV glioma patients $(\mathrm{OR}=1.49$; $95 \% \mathrm{Cl}: 1.03-2.16 ; \mathrm{P}=0.037$ )

Table 2. Frequency of CXCL12 genotypes and alleles in glioma patients and healthy controls.

\begin{tabular}{lrccc}
\hline & Total & Glioma & Control & OR (95\%Cl) \\
\hline Genotype & 1500 & 750 & 750 & \\
GG & 952 & $469(62.53 \%)$ & $483(64.40 \%)$ & $0.968(0.765-1.226)$ \\
AG & 390 & $189(25.20 \%)$ & $201(26.80 \%)$ & $1.436(1.021-2.018)$ \\
AA & 158 & $92(12.27 \%)$ & $66(8.80 \%)$ & \\
Alleles & & & & \\
G & 2294 & $1127(75.13 \%)$ & $333(22.20 \%)$ & $1.160(0.980-1.373)$ \\
A & 706 & $373(24.87 \%)$ & & 0.810 \\
\hline
\end{tabular}

Table 3. Stratification analysis of the CXCL12 G801A polymorphism in glioma (histology).

\begin{tabular}{|c|c|c|c|c|c|c|c|c|c|c|}
\hline \multirow[t]{2}{*}{ Variable } & \multirow[t]{2}{*}{ Glioma } & \multicolumn{3}{|c|}{ GG } & \multicolumn{3}{|c|}{$A G$} & \multicolumn{3}{|c|}{ AA } \\
\hline & & $\mathrm{N}(\%)$ & OR $(95 \% \mathrm{Cl})$ & $P$ value & $\mathrm{N}(\%)$ & OR $(95 \% \mathrm{Cl})$ & $P$ value & $\mathrm{N}(\%)$ & OR $(95 \% \mathrm{Cl})$ & $P$ value \\
\hline Histology & 750 & $469(62.53)$ & & & $189(25.20)$ & & & $92(12.27)$ & & \\
\hline Low-grade & 494 & $322(65.18)$ & $1.04(0.87-1.25)$ & 0.676 & $130(17.33)$ & $1.10(0.86-1.41)$ & 0.478 & $42(8.50)$ & $0.73(0.50-1.07)$ & 0.111 \\
\hline High-grade & 256 & $147(57.42)$ & $0.92(0.73-1.17)$ & 0.478 & 59 (23.05) & $0.92(0.66-1.27)$ & 0.624 & $50(19.53)$ & $1.59(1.10-2.31)$ & 0.019 \\
\hline
\end{tabular}


Table 4. Stratification analysis of the CXCL12 G801A polymorphism in glioma (WHO grade).

\begin{tabular}{|c|c|c|c|c|c|c|c|c|c|c|}
\hline \multirow[t]{2}{*}{ Variable } & \multirow[t]{2}{*}{ Glioma } & \multicolumn{3}{|c|}{ GG } & \multicolumn{3}{|c|}{$A G$} & \multicolumn{3}{|c|}{ AA } \\
\hline & & $\mathrm{N}(\%)$ & OR $(95 \% \mathrm{Cl})$ & $P$ value & $\mathrm{N}(\%)$ & OR $(95 \% \mathrm{Cl})$ & $P$ value & $\mathrm{N}(\%)$ & OR $(95 \% \mathrm{Cl})$ & $P$ value \\
\hline $\mathrm{WHO}$ & 750 & $469(62.53)$ & & & $189(25.20)$ & & & $92(12.27)$ & & \\
\hline I & 156 & $106(67.95)$ & $1.09(0.83-1.43)$ & 0.576 & $39(25.00)$ & $0.99(0.68-1.46)$ & 1.000 & $11(7.05)$ & $0.58(0.30-1.10)$ & 0.095 \\
\hline ॥ & 172 & 109 (63.37) & $1.01(0.78-1.32)$ & 0.946 & $47(27.33)$ & $1.08(0.76-1.55)$ & 0.643 & $16(9.30)$ & $0.76(0.44-1.32)$ & 0.360 \\
\hline III & 143 & $82(57.34)$ & $0.92(0.68-1.23)$ & 0.601 & 47 (32.87) & $1.30(0.90-1.88)$ & 0.171 & $14(9.79)$ & $0.80(0.44-1.44)$ & 0.572 \\
\hline IV & 279 & $172(61.65)$ & $0.99(0.79-1.23)$ & 0.910 & $56(20.07)$ & $0.80(0.57-1.11)$ & 0.196 & $51(18.28)$ & $1.49(1.03-2.16)$ & 0.037 \\
\hline
\end{tabular}

\section{DISCUSSION}

Glioma a disease with high morbidity and mortality, and is generally believed to be contributed by both genetic and environmental factors. However, the mechanisms of glioma formation are unclear. A number of recent studies have indicated that gene polymorphism is closely associated with risk of glioma formation. Xu et al. (2014) showed that the X-ray repair cross complementing group 3 gene (XRCC3) Thr241Met polymorphism increases glioma susceptibility, as its frequency is significantly higher in glioma patients as compared with the control subjects. Retinoblastoma binding protein 6 gene variants were also reported to be associated with glioma, and contribute to glioma susceptibility (Hu et al., 2014). Similarly, CCDC26 gene polymorphism may contribute to glioblastoma susceptibility and progression in the Han Chinese (Wei et al., 2014). Identification of susceptible genetic factors may be beneficial for early diagnosis, predicting clinical outcome and prognosis, or carrying out personalized therapy for glioma patients (Shi et al., 2013). However, currently, only a few biomarkers can be used clinically, therefore, there is a pressing need to find genetic biomarkers for glioma.

CXCL12 is a chemokine of the CXC subfamily that is involved in a broad range of biological processes such as cell migration, proliferation, and differentiation. CXCL12 is considered to play important roles in both HIV and cancer. One of its common receptor CXCR4 is widely expressed in various types of cancer. In addition, CXCL12 and its receptors signaling have been shown to be closely related to proliferation, invasion, metastasis, and angiogenesis of gliomas, suggesting that genetic changes to CXCL12 may be involved in glioma susceptibility. In the present study, our results showed that significant differences in the distribution of the CXCL12-G801A gene variant between patients with glioma and healthy individuals. In addition, CXCL12-G801A genotype was correlated with histological type and tumor grade, suggesting that CXCL12-G801A polymorphism may be a risk factor for glioma.

Consistent with our study, AA and AG genotypes of CXCL12-801 are considered to be factors for increasing the susceptibility of Iranian women to breast and lung cancers (Razmkhah et al., 2005a,b). A significant increase in the GA and AA subgroups was also observed in colorectal cancer patients compared with healthy controls. In addition, frequency of the CXCL12-G801A genotype was vastly increased among older colorectal cancer patients, suggesting that CXCL12G801A may be a risk factor for colorectal cancer in Taiwanese patients. Chang et al. (2014) also found that the CXCL12-G801A polymorphism was associated with local lymph node metastasis in colorectal cancer The frequencies of the AG and AA genotypes of CXCL12 were significantly higher in patients with benign salivary gland tumors as compared with healthy people (Liu et al., 2012). However, no statistically significance was found in the CXCL12-G801A polymorphism distribution was found between oral cancer patients and health control. CXCL12-G801A polymorphism is associated with advanced stages of oral cancer and alcohol abuse (Vairaktaris et al., 2008). Xia 
et al. (2014) conducted a meta-analysis to show that the CXCL12-G801A genotype is a high risk factor for breast carcinoma.

However, various study showed that the CXCL12 G801A polymorphism was not associated with cancer risk. There was no significant difference in the distribution of $A A, A G$, and GG genotypes of CXCL12-801 in patients with transitional cell carcinoma of the bladder and health subjects (Vázquez-Lavista et al., 2008). Furthermore, no difference in the frequency of the CXCL12$801 \mathrm{~A}$ allele was found between malignant salivary gland tumors and controls (Liu et al., 2012). Compared with the homozygous GG subgroup, GA and AA subgroups do not have a higher risk of cervical neoplastic lesions of the uterine cervix (Tee et al., 2012). Similarly, no significant association was found between wild and heterozygous genotypes in clinical manifestations, laboratory data, malignant cell dissemination, and tissue infiltration of patients with acute myeloid leukemias or chronic myeloid leukemias (EI-Ghany et al., 2014). Studies have also found that the CXCL12 G801A polymorphism was not associated with colorectal and gastric cancers in Southern Iranian patients (Razmkhah and Ghaderi, 2013). Lastly, Kruszyna et al. (2010) have demonstrated that the CXCL12-3'A gene variant was not a risk factor for breast cancer incidence in Polish population. These conflicting results regarding the role of CXCL12 G801A polymorphism in cancers may be due to cancer-specific differences as well as ethnic-dependent factors. Larger scale studies are needed to further determine the correlation between CXCL12 G801A polymorphism and glioma risk in other populations.

\section{CONCLUSIONS}

The findings of this study demonstrated that the CXCL12 G801A polymorphism was associated with increased susceptibility to gliomas in a subset of the general Han Chinese population. Because of the importance of CXCL12/ CXCR4 signaling in tumorigenesis, further investigations into the functional role and gene expression of CXCL12 in gliomas are needed.

\section{Conflicts of interest}

The authors declare no conflict of interest.

\section{REFERENCES}

Balkwill F (2003). Chemokine biology in cancer. Semin. Immunol. 15: 49-55.

Bleul CC, Fuhlbrigge RC, Casasnovas JM, Aiuti A, et al. (1999). A highly efficacious lymphocyte chemoattractant, stromal cell derived factor 1 (SDF-1). J. Exp. Med.184: 1101-1109.

Broxmeyer HE, Kim CH, Cooper SH, Hangoc G, et al. (1999). Effects of CC, CXC, C, and CX3C chemokines on proliferation of myeloid progenitor cells, and insights into SDF-1-induced chemotaxis of progenitors. Ann. N. Y. Acad. Sci. 872: 142-162.

Chang SC, Lin PC, Yang SH, Wang HS, et al. (2009). SDF-1alpha G801A polymorphism predicts lymph node metastasis in stage T3 colorectal cancer. Ann. Surg. Oncol. 16: 2323-2330.

do Carmo A, Patricio I, Cruz MT, Carvalheiro H, et al. (2010). CXCL12/CXCR4 promotes motility and proliferation of glioma cells. Ann. Neurosci. 17: 85-86.

Domanska UM, Kruizinga RC, Nagengast WB, Timmer-Bosscha H, et al. (2013). A review on CXCR4/CXCL12 axis in oncology: no place to hide. Eur. J. Cancer 49: 219-30.

El-Ghany HM, El-Saadany ZA, Bahaa NM, Ibrahim NY, et al. (2014). Stromal cell derived factor-1 (CXCL12) chemokine gene variant in myeloid leukemias. Clin. Lab. 60: 735-741.

Feng X, Miao G, Han Y, Xu Y, et al. (2014). Glioma risks associate with genetic polymorphisms of XRCC1 gene in Chinese population. J. Cell. Biochem. 115: 1122-7.

Garcia-Moruja C, Rueda P, Torres C, Alcami J, et al. (2009). Molecular phenotype of CXCL12beta 3'UTR G801A polymorphism 
(rs1801157) associated to HIV-1 disease progression. Curr. HIV Res. 7: 384-389.

Hirata H, Hinoda Y, Kikuno N, Kawamoto K, et al. (2007). CXCL12 G801A polymorphism is a risk factor for sporadic prostate cancer susceptibility. Clin. Cancer Res. 13: 5056-5062.

Hu D, Zhang S, Zhao Y, Wang S, et al. (2014). Association of genetic variants in the retinoblastoma binding protein 6 gene with the risk of glioma: a case-control study in a Chinese Han population. J. Neurosurg. 121: 1209-1218.

Kruszyna $Ł$, Lianeri M, Rubis B, Knula H, et al. (2010). CXCL12-3' G801A polymorphism is not a risk factor for breast cancer. DNA Cell Biol. 29: 423-427.

Liu W, Zhu E, Wang R, Wang L, et al. (2012). CXCL12 G801A polymorphism is associated with an increased risk of benign salivary gland tumors in the Chinese population. Med. Oncol. 29: 677-681.

Razmkhah M and Ghaderi A (2013). SDF-1alpha G801A polymorphism in Southern Iranian patients with colorectal and gastric cancers. Indian J. Gastroenterol. 32: 28-31.

Razmkhah M, Doroudchi M, Ghayumi SM, Erfani N, et al. (2005a). Stromal cell-derived factor-1 (SDF-1) gene and susceptibility of Iranian patients with lung cancer. Lung Cancer 49: 311-315.

Razmkhah M, Talei AR, Doroudchi M, Khalili-Azad T, et al. (2005b). Stromal cell-derived factor-1 (SDF-1) alleles and susceptibility to breast carcinoma. Cancer Lett. 225: 261-266.

Salmaggi A, Gelati M, Pollo B, Marras C, et al. (2005). CXCL12 expression is predictive of a shorter time to tumor progression in low-grade glioma: a single-institution study in 50 patients. J. Neurooncol. 74: 287-293.

Shi MD, Chen JH, Sung HT, Lee JS, et al. (2013). CXCL12-G801A polymorphism modulates risk of colorectal cancer in Taiwan. Arch. Med. Sci. 9: 999-1005.

Tee YT, Yang SF, Wang PH, Tsai HT, et al. (2012). G801A polymorphism of human stromal cell-derived factor 1 gene raises no susceptibility to neoplastic lesions of uterine cervix. Int. J. Gynecol. Cancer. 22: 1297-1302.

Vairaktaris E, Vylliotis A, Spyridonodou S, Derka S, et al. (2008). A DNA polymorphism of stromal-derived factor-1 is associated with advanced stages of oral cancer. Anticancer Res. 28: 271-275.

Vázquez-Lavista LG, Lima G, Gabilondo F and Llorente L (2009). Genetic association of monocyte chemoattractant protein 1 (MCP-1)-2518 polymorphism in Mexican patients with transitional cell carcinoma of the bladder. Urology 74: 414-418.

Wei XB, Jin TB, Li G, Geng TT, et al. (2014). CCDC26 gene polymorphism and glioblastoma risk in the Han Chinese population. Asian Pac. J. Cancer Prev. 15: 3629-3633.

Winkler C, Modi W, Smith MW, Wu X, et al. (1998). Genetic restriction of AIDS pathogenesis by an SDF-1 chemokine gene variant. Science 279: 389-393

Xia Y, Guo XG and Ji TX (2014). The G801A polymorphism in the CXCL12 gene and risk of breast carcinoma: evidence from a meta-analysis including 2,931 subjects. Asian Pac. J. Cancer Prev. 15: 2857-2861.

Xu G, Wang M, Xie W and Bai X (2014). DNA repair gene XRCC3 Thr241Met polymorphism and susceptibility to glioma: A case-control study. Oncol Lett. 8: 864-868.

Zhang J, Sarkar S and Yong VW (2005). The chemokine stromal cell derived factor-1 (CXCL12) promotes glioma invasiveness through MT2-matrix metalloproteinase. Carcinogenesis 26: 2069-2077. 